This is a self-archived version of an original article. This version may differ from the original in pagination and typographic details.

Author(s): Aikebaier, Faluke; Silaev, Mikhail; Heikkilä, Tero

Title: Supercurrent-induced charge-spin conversion in spin-split superconductors

Year: 2018

Version: Published version

Copyright: @ 2018 American Physical Society

Rights: In Copyright

Rights url: http://rightsstatements.org/page/lnC/1.0/?language=en

Please cite the original version:

Aikebaier, F., Silaev, M., \& Heikkilä, T. (2018). Supercurrent-induced charge-spin conversion in spin-split superconductors. Physical Review B, 98(2), Article 024516.

https://doi.org/10.1103/physrevb.98.024516 


\title{
Supercurrent-induced charge-spin conversion in spin-split superconductors
}

\author{
Faluke Aikebaier, ${ }^{*}$ Mihail A. Silaev, ${ }^{\dagger}$ and T. T. Heikkilä ${ }^{\ddagger}$ \\ Department of Physics and Nanoscience Center, University of Jyvaskyla, P.O. Box 35 (YFL), FI-40014 University of Jyväskylä, Finland
}

(Received 22 December 2017; revised manuscript received 27 March 2018; published 25 July 2018)

\begin{abstract}
We study spin-polarized quasiparticle transport in a mesoscopic superconductor with a spin-splitting field in the presence of coflowing supercurrent. In such a system, the nonequilibrium state is characterized by charge, spin, energy, and spin-energy modes. Here we show that in the presence of both spin splitting and supercurrent, all these modes are mutually coupled. As a result, the supercurrent can convert charge imbalance, which in the presence of spin splitting decays on a relatively short scale, to a long-range spin accumulation decaying only via inelastic scattering. This effect enables coherent charge-spin conversion controllable by a magnetic flux, and it can be detected by studying different symmetry components of the nonlocal conductance signal.
\end{abstract}

DOI: 10.1103/PhysRevB.98.024516

\section{INTRODUCTION}

The nonequilibrium states in superconductors can be classified in terms of energy and charge modes [1,2] as a direct result of the particle-hole formalism in BCS theory. In magnetic systems the relevant nonequilibrium modes are related to the quasiparticle spin. In spin-split superconductors all these modes need to be considered, and the quasiparticle diffusion couples pairs of modes [3-5]. The earlier description of such spin-resolved modes includes only the direct quasiparticle transport, whereas the effect of supercurrent was not considered. However, a supercurrent flowing along a temperature gradient is known to induce a charge imbalance [6-9]. Here we combine these two effects and show how supercurrent couples all nonequilibrium modes. We show how this leads to a large coherently controllable charge-spin conversion induced by supercurrent. In particular, we use the theoretical framework [3] based on the quasiclassical Keldysh-Usadel formalism for superconductors with a spin-splitting field $h$ and consider the presence of a constant phase gradient $\nabla \varphi$ in the superconducting order parameter. This leads to supercurrent and shows up in the kinetic equations as spectral charge and spin supercurrents. These coherent supercurrent terms couple spin and charge transport, generating spin from charge injection. The effect is long ranged compared to the spin-relaxation length in the normal state and becomes very large at the critical temperature and exchange field. It can be detected by studying the different symmetry components of the nonlocal conductance.

The spin-charge conversion studied here occurs only under nonequilibrium conditions and does not require spin-orbit interaction. Therefore it is qualitatively different from the direct [10-12] and inverse [13-16] equilibrium magnetoelectric effects proposed for noncentrosymmetric superconductors, Josephson junctions [17-19], and superconducting hybrid systems [20] with spin-orbit coupling. Experimental

\footnotetext{
*faluke.aikebaier@jyu.fi

†mikesilaev@gmail.com

†tero.t.heikkila@jyu.fi
}

verification of these spin-orbit-induced effects is limited to recent observations of the anomalous Josephson effect through a quantum dot [21] and $\mathrm{Bi}_{2} \mathrm{Se}_{3}$ interlayer [22,23]. To our knowledge, the direct magnetoelectric effect, also known as the Edelstein effect, in noncentrosymmetric superconductors has not been observed to date. In normal conductors, such as GaAs semiconductors, this effect is known as the inverse spingalvanic effect and has been detected using Faraday rotation [24]. In contrast, the charge-spin conversion predicted in this work can be measured by purely electrical probes. Moreover, it is specific to superconducting metallic systems and does not rely on the combination of inversion symmetry breaking and spin-orbit coupling, which usually has a tiny effect in such materials.

\section{QUALITATIVE DESCRIPTION OF THE CHARGE-SPIN CONVERSION}

The supercurrent-generated coupling between different nonequilibrium states can be understood with the schematic in Fig. 1, showing the spin-split BCS spectrum $E_{p}+\sigma h \pm \boldsymbol{p}_{F} \boldsymbol{v}_{s}$ (where $\sigma= \pm 1$ for spin $\uparrow$ and $\downarrow$ ) for left- and right-moving quasiparticles with respect to the condensate velocity $\boldsymbol{v}_{s}$. The left- and right-moving states are defined according to their velocities $v_{g} \equiv \partial E_{p} / \partial \boldsymbol{p} \gtrless 0$. The balance between the two can be broken either by position-dependent nonequilibrium modes or by the presence of a supercurrent that induces an energy difference (Doppler shift) $\sim 2 p_{F} v_{s}$ between the states with $p \approx \pm p_{F}$, where $p_{F}$ is the Fermi momentum.

In the absence of spin splitting, $h=0$, the combination of these two effects allows for the creation of charge imbalance proportional to $\boldsymbol{v}_{s} \boldsymbol{\nabla} T$ [6-9] where $T$ is the temperature. This mechanism is illustrated qualitatively in Fig. 1(a). Due to the temperature gradient, left-moving quasiparticles [both electrons (el) and holes (hl)] with velocities $v_{e}=v_{h}=-v_{g}=$ $-v_{F} \sqrt{E_{p}^{2}-\Delta^{2}} / E_{p}$ have an excess temperature $T_{L}$ compared to that of the right-moving particles $T_{R}$. From Fig. 1(a) one can see that due to the Doppler shift there are more occupied states in the electron branch. This results in the charge imbalance $\mu$ controlled by the Doppler shift $p_{F} v_{s}$. 

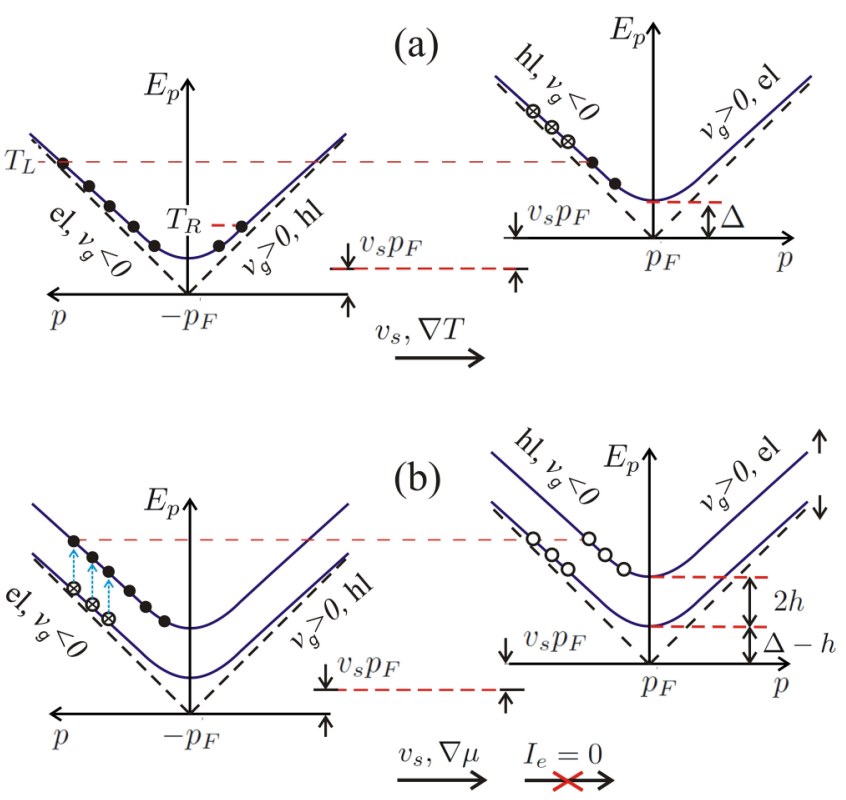

FIG. 1. Schematic pictures illustrating the couplings between different types of nonequilibrium states in a superconductor in the presence of the phase gradient driving the condensate to the velocity $\boldsymbol{v}_{s}$. (a) Generation of charge imbalance by the temperature gradient. (b) Generation of spin accumulation by the charge imbalance gradient $\nabla \mu$ under the restriction that energy current is absent $I_{e}=0$. Shown in the plots are the quasiparticle electronlike (el) and holelike (hl) spectral branches in the superconductor in the presence of Doppler shifted energy $\pm p_{F} v_{s}$. The solid and open circles show the extra occupied and empty states compared to the equilibrium distribution, respectively, and the circles with crosses show the states which become depopulated due to the Doppler shift.

Now, let us turn to the system in the presence of velocity $v_{s}$ and Zeeman splitting $h \neq 0$, shown in Fig. 1(b). Spin splitting the spectrum provides the possibility for a population difference between spin- $\uparrow$ and $-\downarrow$ branches. Therefore the supercurrent can couple charge and spin $\left(\mu_{z} \propto \boldsymbol{v}_{s} \nabla \mu\right.$ or $\left.\mu \propto \boldsymbol{v}_{s} \nabla \mu_{z}\right)$ as well as excess energy and spin energy $\left(T_{s} \propto \boldsymbol{v}_{s} \boldsymbol{\nabla} T\right.$ or $\left.T \propto \boldsymbol{v}_{s} \boldsymbol{\nabla} T_{s}\right)$. Here $\mu_{z}$ is the spin accumulation, and $T_{s}$ is the spin energy accumulation [3]. Under general nonequilibrium conditions all these couplings are present. To separate the particular charge-spin conversion effect we must impose certain constraints on the distribution function changes due to the supercurrent-induced Doppler shift as in Fig. 1(b). As shown below [Eq. (18)], these constraints determine the particular symmetry components of the nonlocal conductance as functions of the injector voltage and polarization of the detector electrode. For example, let us assume a charge imbalance gradient $\nabla \mu \neq 0$ resulting in a larger (smaller) number of leftmoving electrons (holes) in the absence of energy current $I_{e}$, so that the energies of left-moving (right-moving) quasiparticles are the same. In the absence of supercurrent these states occupy spin-up and -down branches symmetrically, yielding no spin accumulation. The Doppler shift results in qualitative changes in quasiparticle distributions. From Fig. 1(b) one can see that in order to have $I_{e}=0$ without affecting the charge imbalance, the extra energy gained by placing electrons on the Doppler-shifted energy branch can be compensated only by utilizing the Zeeman energy and shifting some occupied states on the spin-down electron branch to the spin-up one (dashed arrows in Fig. 1). Together with compensating the energy difference between left- and right-moving states this shift produces a net spin polarization.

\section{KINETIC THEORY IN THE PRESENCE OF SUPERCURRENT AND SPIN SPLITTING}

Below, we quantify the physics described above using the kinetic equations [3] based on the quasiclassical KeldyshUsadel formalism for superconductors with a spin-splitting field $h$ to study the spin accumulation generated by the charge imbalance gradients. For concreteness, we consider the structure shown in Fig. 3(a) below. A superconducting wire with length $L$ is placed between two superconducting reservoirs. We assume the presence of a Zeeman splitting along the wire due to either a magnetic proximity effect from a ferromagnetic insulator or an in-plane magnetic field. A current is injected in the wire from a normal-metal injector. A ferromagnetic detector with normal-state conductance $G_{\text {det }}$ and spin polarization $P_{\text {det }}$ is placed at distance $L_{\text {det }}$ from the injector. Variants of this setup were realized, for example, in Refs. [25-27]. Here we assume that, in addition, a homogeneous supercurrent $I_{s}$ flows along the wire. Either this current can be driven externally, or it can be induced by a magnetic field in a superconducting loop.

To study the properties of a mesoscopic superconductor with Zeeman splitting, we start from the Usadel equation [28] $\left(\hbar=k_{B}=1\right)$

$$
D \hat{\nabla}(\check{g} \hat{\nabla} \check{g})+\left[\check{\Lambda}-\check{\Sigma}_{\mathrm{so}}-\check{\Sigma}_{\mathrm{sf}}-\check{\Sigma}_{\text {orb }}, \check{g}\right]=0,
$$

where $D$ is the diffusion constant, $\check{g}$ is the quasiclassical Green's function, and the covariant gradient operator is $\hat{\nabla}=$ $\boldsymbol{\nabla}-i \boldsymbol{A}\left[\tau_{3}, \cdot\right]$. In the commutator $\check{\Lambda}=i \in \tau_{3}-i(\boldsymbol{h} \cdot \boldsymbol{S}) \tau_{3}-\check{\Delta}$, $\epsilon$ is the quasiparticle energy, $\boldsymbol{h}$ is the spin-splitting field, $\boldsymbol{S}=\left(\sigma_{1}, \sigma_{2}, \sigma_{3}\right)$, and the Pauli matrix $\tau_{j}\left(\sigma_{j}\right)$ is in Nambu (spin) space. The exact form of the spin-splitting field term, as well as of the pair potential $\hat{\Delta}$, depends on the chosen Nambu spinor. We choose it to be

$$
\Psi=\left(\psi_{\uparrow}(x), \psi_{\downarrow}(x),-\psi_{\downarrow}^{\dagger}(x), \psi_{\uparrow}^{\dagger}(x)\right)^{T},
$$

where $T$ denotes a transpose. The advantage of using this spinor is that the Nambu structure has the same form for each spin component. The superconducting pair potential $\check{\Delta}=$ $\hat{\Delta} \sigma_{0}$ should be obtained self-consistently (see Appendix A for details). We denote the Nambu-space matrix $\hat{\Delta}(x)=$ $|\Delta| e^{i \varphi(x) \tau_{3}} \tau_{1}$, where $x$ is the coordinate along the wire. Due to supercurrent, the phase $\varphi$ becomes position dependent. We assume that the quasiparticle currents within the wire are so small that we can disregard the ensuing position dependence of $|\Delta|$. The last three terms in the commutator are $\check{\Sigma}_{\text {so }}=\left(8 \tau_{\text {so }}\right)^{-1}(\boldsymbol{S} \check{g} \boldsymbol{S}), \check{\Sigma}_{\text {sf }}=\left(8 \tau_{\text {sf }}\right)^{-1}\left(\boldsymbol{S} \tau_{3} \check{g} \tau_{3} \boldsymbol{S}\right)$, and $\check{\Sigma}_{\text {orb }}=$ $\tau_{\text {orb }}^{-1} \tau_{3} \check{g} \tau_{3}$, representing spin and charge imbalance relaxation due to the spin-orbit scattering, exchange interaction with magnetic impurities, and orbital magnetic depairing, respectively. The corresponding relaxation rates are $\tau_{\mathrm{so}}^{-1}, \tau_{\mathrm{sf}}^{-1}$, and $\tau_{\mathrm{orb}}^{-1}$. 
We use the real-time Keldysh formalism and describe the quasiclassical Green's function as

$$
\check{g}=\left(\begin{array}{cc}
\hat{g}^{R} & \hat{g}^{K} \\
\hat{0} & \hat{g}^{A}
\end{array}\right),
$$

where each component is a $4 \times 4$ matrix in the Nambu $\otimes$ spin space, $\hat{g}^{R(A)}$ is the retarded (advanced) Green's function, and $\hat{g}^{K}$ is the Keldysh Green's function describing the nonequilibrium properties. This function can be parameterized in the case of collinear magnetizations by $\hat{g}^{K}=\hat{g}^{R} \hat{f}-\hat{f} \hat{g}^{A}$, where the distribution matrix $\hat{f}=f_{L}+f_{T} \tau_{3}+f_{T 3} \sigma_{3}+f_{L 3} \sigma_{3} \tau_{3}$.

We consider Eq. (1) in the presence of the superconducting current along the wire. Removing the phase of the order parameter by gauge transformation allows us to write Eq. (1) in the gauge-invariant form, replacing the vector potential by the condensate momentum $\boldsymbol{q}_{s}=\nabla \varphi-2 A$. The gradient term in Eq. (1) can be written in the form

$$
\begin{gathered}
\hat{\boldsymbol{\nabla}} \cdot(\check{g} \hat{\nabla} \breve{g})=\nabla \cdot \hat{\boldsymbol{I}}+\frac{i}{2}\left[\tau_{3}, \boldsymbol{q}_{s} \hat{\boldsymbol{I}}\right], \\
\hat{\boldsymbol{I}}=\check{g} \nabla \check{g}+\frac{i \boldsymbol{q}_{s}}{2}\left(\check{g} \tau_{3} \check{g}-i \tau_{3}\right),
\end{gathered}
$$

where $\hat{\boldsymbol{I}}$ is the matrix spectral current. We formulate the Keldysh part of this equation in terms of spectral currents: charge $j_{c}=\operatorname{Tr}\left(\tau_{3} \hat{I}\right)$, energy $j_{e}=\operatorname{Tr}\left(\tau_{0} \hat{I}\right)$, spin $j_{s}=\operatorname{Tr}\left(\sigma_{3} \hat{I}\right)$, and spin energy $j_{\text {se }}=\operatorname{Tr}\left(\sigma_{3} \tau_{3} \hat{I}\right)$.

Kinetic equations derived from Eqs. (4) and (5) for these currents can be written in a matrix form:

$$
\nabla \cdot\left(\begin{array}{c}
j_{e} \\
j_{s} \\
j_{c} \\
j_{\mathrm{se}}
\end{array}\right)=\left(\begin{array}{cccc}
0 & 0 & 0 & 0 \\
0 & S_{T 3} & 0 & 0 \\
0 & 0 & R_{T} & R_{L 3} \\
0 & 0 & R_{L 3} & R_{T}+S_{L 3}
\end{array}\right)\left(\begin{array}{c}
f_{L} \\
f_{T 3} \\
f_{T} \\
f_{L 3}
\end{array}\right)
$$

where

$$
\left(\begin{array}{c}
j_{e} \\
j_{s} \\
j_{c} \\
j_{\mathrm{se}}
\end{array}\right)=\left(\begin{array}{cccc}
D_{L} \nabla & D_{T 3} \nabla & j_{E} q_{s} & j_{E s} q_{s} \\
D_{T 3} \nabla & D_{L} \nabla & j_{E s} q_{s} & j_{E} q_{s} \\
j_{E} q_{s} & j_{E s} q_{s} & D_{T} \nabla & D_{L 3} \nabla \\
j_{E s} q_{s} & j_{E} q_{s} & D_{L 3} \nabla & D_{T} \nabla
\end{array}\right)\left(\begin{array}{c}
f_{L} \\
f_{T 3} \\
f_{T} \\
f_{L 3}
\end{array}\right) .
$$

The kinetic coefficients $D_{L / T / T 3 / L 3}, R_{T / L 3}$, and $S_{T 3 / L 3}$ are defined in terms of the components of $\hat{g}^{R}$ and $\hat{g}^{A}$ (see Appendix B and more details in Ref. [3]). The terms $S_{T 3 / L 3}$ are proportional to the total spin-relaxation rate in the normal state, $\tau_{\mathrm{sn}}^{-1}=$ $\tau_{\mathrm{so}}^{-1}+\tau_{\mathrm{sf}}^{-1}$. The phase gradient provides two additional terms in Eq. (7): spectral supercurrent $j_{E}$ [29] and spin supercurrent $j_{E s}=D \operatorname{Tr}\left[\left(\hat{g}^{R} \nabla \hat{g}^{R}-\hat{g}^{A} \nabla \hat{g}^{A}\right) \sigma_{3} \tau_{3}\right] /\left(8 q_{s}\right)$. Further we use the gauge with $\boldsymbol{A}=0$, so that $\boldsymbol{q}_{s}=\nabla \varphi$.

In equilibrium $f_{L}=\tanh (\epsilon / 2 T) \equiv n_{0}$, and other modes are absent. Then the spectral current terms yield nonzero charge supercurrent $I_{s}$ and spin-energy current $I_{\mathrm{se}}$ as

$$
\begin{gathered}
I_{s}=G_{\xi_{0}} \xi_{0} q_{s} \int_{-\infty}^{\infty} d \epsilon j_{E} \tanh \left(\frac{\epsilon}{2 T}\right), \\
I_{\mathrm{se}}=G_{\xi_{0}} \xi_{0} q_{s} \int_{-\infty}^{\infty} d \epsilon \epsilon j_{E s} \tanh \left(\frac{\epsilon}{2 T}\right),
\end{gathered}
$$

where $G_{\xi_{0}}=e^{2} D v_{F} A / \xi_{0}$ is the normal-state conductance of the wire of one superconducting coherence length

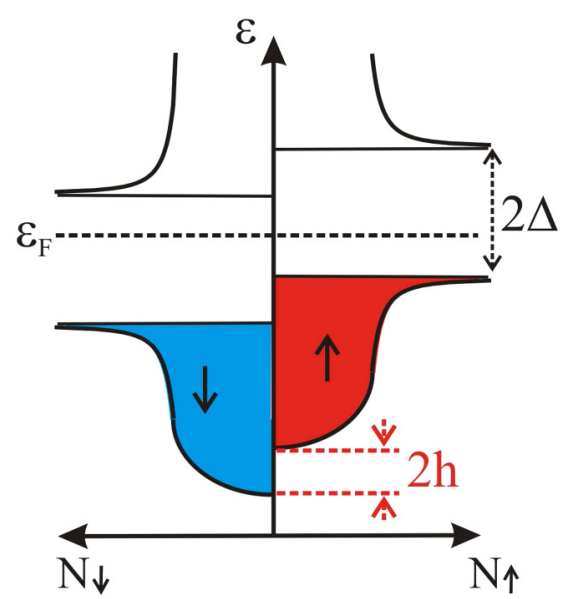

FIG. 2. Schematic picture illustrating the nonzero spin energy in the ground state of a spin-singlet superconductor with spin splitting. $N_{\uparrow, \downarrow}(\varepsilon)$ are the spin-up and -down densities of states as functions of the energy $\varepsilon$. The relative Zeeman shift of the electronic bands is $2 h$. The case of $T=0$ is shown, so that all states below the Fermi level $\varepsilon_{F}$ are occupied.

$\xi_{0}=\sqrt{D / \Delta}$, with normal-state density of states $v_{F}$ and cross section $A$. We assume that the phase gradient is small, so that $I_{s}$ is much below the critical current of the wire.

The equilibrium spin-energy current, Eq. (9), arises due to the modification of the superconducting ground state in the presence of an exchange field. This is illustrated schematically in Fig. 2, which shows the occupied energy states in spin-up and spin-down subbands in a superconductor with a spin-splitting field. Here one can see that there is a relative energy shift between the spin-up and -down subbands. The overall energy difference between these states yields the nonvanishing spin energy density $\epsilon_{\uparrow}-\epsilon_{\downarrow}=h N_{0}$, where $N_{0}$ is the total electron density. Since all these particles are in the condensed state, the collective motion of the condensate results in the coherent spinenergy flow $I_{\mathrm{se}}=v_{s} N_{0} h$. However, such an equilibrium spinenergy current is not directly observable and can be revealed through its coupling to the superconducting current and charge imbalance, as discussed below.

Out of equilibrium, the matrix in Eq. (7) couples the four modes together. The diffusion coefficients $D_{T 3 / L 3} \neq 0$ for $h \neq 0$ combine pairwise $f_{T}$ and $f_{L 3}$ (charge and spin energy) modes as well as $f_{L}$ and $f_{T 3}$ (energy and spin) modes [4,5]. An additional coupling between $f_{L}$ and $f_{T}$ modes is introduced by $j_{E}$, mixing charge imbalance with energy. This coupling leads to the supercurrent-induced charge imbalance in the presence of a temperature gradient [7-9]. The presence of $h$ and $j_{E}$ combines these two effects together in Eq. (7) and allows for the conversion between charge imbalance and spin accumulation. In the next section we study the observable consequences of this conversion.

\section{SPIN-CHARGE CONVERSION IN A NONLOCAL SPIN VALVE}

Kinetic theory developed in the previous section can be applied to predict the experimentally measurable consequence of the charge-spin conversion effect in the nonlocal spin-valve 

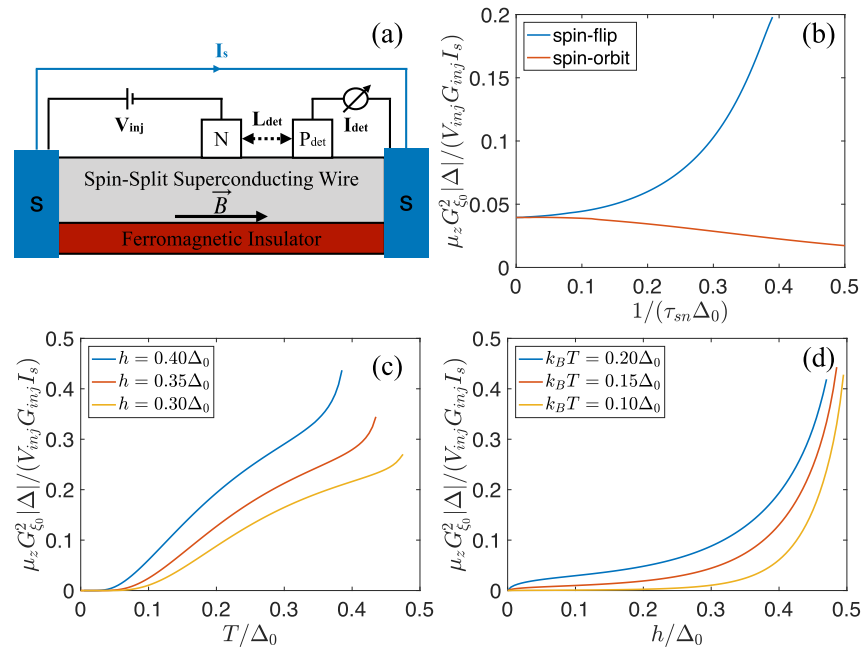

FIG. 3. (a) Schematic view of the setup. Here the spin-splitting field is induced from either the ferromagnetic insulator or external magnetic field $\boldsymbol{B}$. (b)-(d) Spin accumulation as a function of parameters $\mu_{z}=\mu_{z}\left(h, T, \tau_{\mathrm{sn}}\right)$ at the detector position $L_{\mathrm{det}}=L / 8$ in the linear-response regime (small $V_{\text {inj }}$ ). (b) The dependence on the spinrelaxation rate for $k_{B} T=0.15 \Delta_{0}$ and $h=0.3 \Delta_{0}$. (c) Temperature and (d) spin-splitting field dependence. The orbital depairing rate is $\tau_{\text {orb }}^{-1}=0.176 h^{2} / \Delta_{0}$. Here we normalize the induced spin signal by the supercurrent amplitude $I_{s}$

setup shown in Fig. 3(a). It consists of a superconducting wire with externally induced supercurrent, an injector electrode attached at $x=0$, and a ferromagnetic detector electrode attached at some distance $x=L_{D}$. The overall length of the wire $L$ is fixed by the boundary conditions which require all nonequilibrium modes to vanish at $x= \pm L / 2$.

Consider a nonferromagnetic injector electrode attached at $x=0$. We describe the injection of matrix quasiparticle current using the boundary conditions at the tunneling interface [30] extended to the spin-dependent case [31]:

$$
\left(\begin{array}{c}
{\left[j_{c}\right]} \\
{\left[j_{e}\right]} \\
{\left[j_{s}\right]} \\
{\left[j_{\mathrm{se}}\right]}
\end{array}\right)=\left(\begin{array}{cccc}
N_{+} & P N_{-} & P N_{+} & N_{-} \\
P N_{-} & N_{+} & N_{-} & P N_{+} \\
P N_{+} & N_{-} & N_{+} & P N_{-} \\
N_{-} & P N_{+} & P N_{-} & N_{+}
\end{array}\right)\left(\begin{array}{c}
{\left[f_{T}\right]} \\
{\left[f_{L}\right]} \\
{\left[f_{T 3}\right]} \\
{\left[f_{L 3}\right]}
\end{array}\right) .
$$

Here the left-hand side of Eq. (10) contains the differences between currents in the superconducting wire on the left and on the right from the injector, $\left[j_{k}\right]=\left[j_{k}(x=+0)-j_{k}(x=\right.$ $-0)] / \kappa_{I}$, where $k=T, L, T 3, L 3$ and $\kappa_{I}=G_{\text {inj }} /\left(G_{L} L\right)$ is the injector transparency defined by the ratio of the normal-state conductance $G_{\text {inj }}$ of the injector and the conductance $G_{L} L$ of the wire per unit length.

The right-hand side of Eq. (10) contains the differences of the distribution function components $[f]_{k}=f_{k}^{(S)}-f_{k}^{(N)}$ between the superconductor and normal-metal electrodes. The response matrix is here described by the spin polarization $P$ and the energy-symmetric and energy-antisymmetric parts of the density of states, $N_{+}=\operatorname{Tr} \operatorname{Re}\left(\tau_{3} \hat{g}^{R}\right)$ and $N_{-}=$ $\operatorname{Tr} \operatorname{Re}\left(\sigma_{3} \tau_{3} \hat{g}^{R}\right)$. In our particular case the normal-metal injector is characterized by the Fermi distribution function shifted by the applied bias voltage $V_{\text {inj. }}$. Therefore we have $\left[f_{L}\right]=$
$f_{L}-n_{+},\left[f_{T}\right]=\left(f_{T}-n_{-}\right),\left[f_{T 3}\right]=f_{T 3}$, and $\left[f_{L 3}\right]=f_{L 3}$, where $n_{ \pm}=\left[n_{0}\left(\epsilon+V_{\text {inj }}\right) \pm n_{0}\left(\epsilon-V_{\text {inj }}\right)\right] / 2$.

The solutions of Eqs. (6) and (10) can be used to calculate the tunneling current $I_{\text {det }}$ measured by a spin-polarized detector [4] with spin-filtering efficiency $P_{\text {det }}$ :

$$
\begin{gathered}
I_{\mathrm{det}}=G_{\mathrm{det}}\left(\mu+P_{\mathrm{det}} \mu_{z}\right), \\
\mu=\frac{1}{2} \int_{-\infty}^{\infty} d \varepsilon\left(N_{+} f_{T}+N_{-} f_{L 3}\right), \\
\mu_{z}=\frac{1}{2} \int_{-\infty}^{\infty} d \varepsilon\left[N_{+} f_{T 3}+N_{-}\left(f_{L}-f_{\mathrm{eq}}\right)\right] .
\end{gathered}
$$

The contributions from the different nonequilibrium modes to $\mu$ and $\mu_{z}$ can be read off from the different symmetry components of $I_{\mathrm{det}}$ with respect to the injection voltage $V_{\mathrm{inj}}$ and the detector polarization $P_{\text {det }}$. The non-spin-polarized injector generates charge $f_{T}$ and energy $f_{L}$ modes [32], which are odd and even in the injection voltage, respectively. In spin-split superconductors the energy mode is coupled to the spin accumulation, producing a long-range spin signal with the symmetry [4] $\mu_{z}\left(V_{\text {inj }}\right)=\mu_{z}\left(-V_{\text {inj }}\right)$. The supercurrent converts part of the charge imbalance to long-range spin accumulation with the opposite symmetry $\mu_{z}\left(V_{\text {inj }}\right)=-\mu_{z}\left(-V_{\text {inj }}\right)$. Below we concentrate on the details of this mechanism.

First, we solve the kinetic equations using a perturbation expansion in the small parameter $\xi_{0} q_{s}$, where $\xi_{0}=\sqrt{D / \Delta}$ is the coherence length. For simplicity, we disregard inelastic scattering that would add an energy-nonlocal term in Eq. (6) and rather assume that $f_{L}=n_{0}$ at the ends of the wire. This mimics the typical experimental situation where the wire ends in wide electrodes, often at a distance that is small compared to the inelastic scattering length. In this case the solution of $f_{L}$ includes a linear component. The solution of $f_{T 3}$, however, is determined by the strength of spin relaxation. This calculation is detailed in Appendix C.

When $q_{s}=0$, we find $f_{T}$ and $f_{L 3}$ modes generating the charge imbalance $\mu$. For $q_{s} \neq 0$ [see Eq. (7)] these solutions provide sources for the $f_{L}$ and $f_{T 3}$ modes generating the spin accumulation $\mu_{z}$ in accordance with the qualitative mechanism illustrated in Fig. 1(b). This generation takes place close to the injectors before the charge imbalance relaxes due to the presence of an exchange field and depairing $[3,33]$ [blue lines in Fig. 4(a)].

However, $\mu_{z}$ has a long-range part associated with the contribution of $f_{L}$, which consists of two qualitatively different parts. First, even in the absence of the supercurrent, there exists a long-range contribution related to the already known heating effect [4] given by

$$
f_{L}^{\text {heat }}(x)=\alpha_{\text {heat }}(|x|-L),
$$

where $\alpha_{\text {heat }}=N_{+} n_{+} / D_{L}$. Second, the long-range contribution excited due to the supercurrent is given approximatively by

$$
f_{L}^{\text {super }}=\alpha_{\text {super }}[\operatorname{sgn}(x)-x / L] .
$$

The amplitude $\alpha_{\text {super }}$ depends on the strength of relaxation described by $R_{T / L 3}$ and $S_{T 3 / L 3}$ in Eq. (6).

Note that the spatial structures of (14) and (15) are different because $f_{L}^{\text {heat }}(x)$ is an even function and $f_{L}^{\text {super }}(x)$ is an odd function of $x$ [see Fig. 4(a)]. In addition, the amplitude of 
(a)

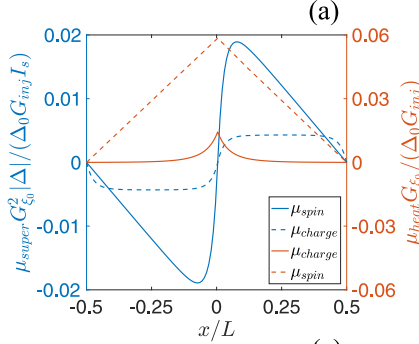

(c)

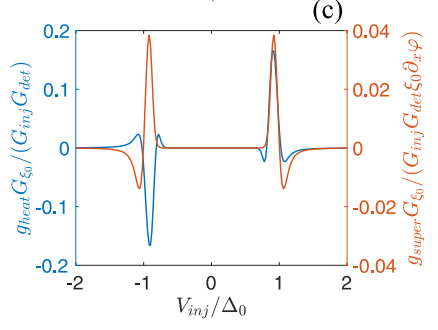

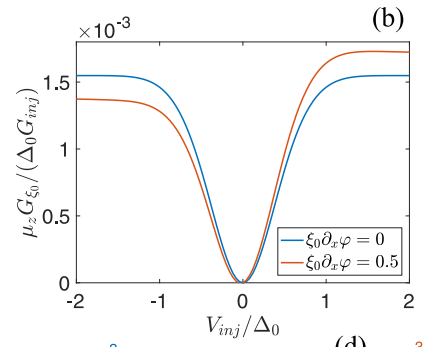

(d) $\times 3^{10^{-3}}$

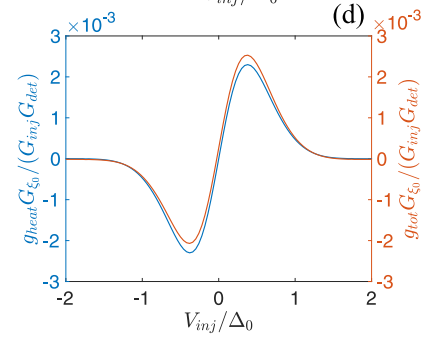

FIG. 4. Spin accumulation and nonlocal conductance. (a) Position dependence of heat-induced (red) and supercurrent-induced (blue) charge and spin imbalances. Here the results are calculated for $T=0.15 \Delta_{0}, h=0.3 \Delta_{0}$ at $V_{\text {inj }}=0.1 \Delta_{0}$. The solid curves are odd in injection voltage, and dashed curves are even. (b) Injection voltage dependence on spin accumulation for $T=0.25 \Delta_{0}$. (c) Nonlocal conductance as a function of injection voltage in separate scales for heat- and supercurrent-induced effects (with $\xi_{0} \partial_{x} \varphi=0.1$ ) for $T=0.02 \Delta_{0}$. (d) Heat-induced and total conductance as a function of injection voltage for $T=0.25 \Delta_{0}$ (with $\xi_{0} \partial_{x} \varphi=0.5$ ). The parameters $\tau_{\text {so }}^{-1}=0.0475 \Delta_{0}, \tau_{\text {sf }}^{-1}=0.0025 \Delta_{0}, h=0.05 \Delta_{0}$, and $L=20 \xi_{0}$ are the same in (b)-(d).

the supercurrent-induced part is an odd function of the injector voltage $\alpha_{\text {super }}\left(V_{\text {inj }}\right)=-\alpha_{\text {super }}\left(-V_{\text {inj }}\right)$. Therefore it exists already in the linear regime, whereas the heating (14) is a nonlinear effect since $\alpha_{\text {heat }}\left(V_{\text {inj }}\right)=\alpha_{\text {heat }}\left(-V_{\text {inj }}\right)$. Further, as one can see from Eq. (14), the heating contribution grows linearly with the wire length $L$, while the supercurrent-related part (15) does not depend on the length $L$ at distances $|x| \ll L$.

To gain further insight, we first study the spin accumulation using a numerical solution of the kinetic equations. In Figs. 3(b)-3(d), 4(a), and 4(b), we show the dependencies of the spin accumulation on various parameters $\mu_{z}=$ $\mu_{z}\left(h, T, \tau_{\mathrm{sn}}, V_{\mathrm{inj}}, x\right)$ obtained from the numerical solutions of Eqs. (6) and (7). Note that from this plot it is clear that the effect exists entirely due to the modification of the quasiparticle spectrum by the spin splitting: As shown in Figs. 3(c) and 3(d), the spin signal $\mu_{z}$ disappears both for $h \rightarrow 0$ when there is no spin splitting and for $T \rightarrow 0$ when there are no quasiparticles. At the same time, Fig. 3(b) shows that the effect survives in the absence of spin-orbit or spin-flip scattering, i.e., for $\tau_{\mathrm{sn}} \rightarrow \infty$. Below we study in more detail the influence of spin relaxation on the behavior of different contributions to the spin accumulation.

\section{A. Case without spin relaxation $\left(S_{T 3, L 3}=0\right)$}

The discussed mechanism of spin-charge conversion does not require any nonconservation of spin. This is qualitative distinction from previously discussed direct and inverse Edelstein effects which rely on the spin-orbit interaction [1016]. In the absence of spin relaxation, $f_{T 3} \propto x$ is also a

long-range mode similar to the longitudinal one which in the absence of inelastic scattering is long range [see Eqs. (14) and (15)]. The combination of $f_{T 3}$ and $f_{L}$ then yields (see details in Appendix C)

$$
\mu_{z}=\xi_{0} \partial_{x} \varphi \frac{G_{\mathrm{inj}}}{G_{\xi_{0}}} \int_{0}^{\infty} d \epsilon n_{-}\left(\epsilon ; V_{\mathrm{inj}}\right) \sum_{\sigma=\uparrow, \downarrow} \frac{\sigma N_{\sigma}^{2} j_{s}^{\sigma}}{4 D_{L}^{\sigma} R_{T}^{\sigma}} u_{0}(x) .
$$

Here $u_{0}(x)=-u_{0}(-x)$ is a function that decays linearly from unity close to the injector $(x=0)$ to zero at the reservoirs, and $n_{-}=\left[n_{0}\left(\epsilon+V_{\text {inj }}\right)-n_{0}\left(\epsilon-V_{\text {inj }}\right)\right] / 2$. Equation (16) describes the region $|x|>\lambda_{\text {cr }}$, where $\lambda_{\text {cr }}$ is the charge relaxation length. Here $N_{\uparrow / \downarrow}=N_{+} \pm N_{-}$are spin-up and -down densities of states, $D_{L}^{\uparrow / \downarrow}=D_{L} \pm D_{T 3}, R_{T}^{\uparrow / \downarrow}=R_{T} \pm R_{L 3}$, and $j_{s}^{\uparrow / \downarrow}=$ $j_{E} \pm j_{E s}$. Moreover, $G_{\mathrm{inj}}$ and $G_{\xi_{0}}$ are the normal-state conductances of the injector and of a wire with length $\xi_{0}$, respectively. The integrand in Eq. (16) is peaked at $\epsilon \approx \Delta \pm h$ due to the BCS divergence in $N_{\sigma}, j_{s}^{\sigma}$, and $R_{T}^{\sigma}$. This divergence can be cut off by the depairing parameter [34] $\Gamma$. Then taking $\epsilon=$ $\Delta+h$ for spin-up $\sigma=\uparrow$ and $\epsilon=\Delta-h$ for spin-down $\sigma=\downarrow$ we obtain, $N_{\sigma} \approx \gamma_{\sigma}^{-1 / 2} / \sqrt{2}, j_{S}^{\sigma} \approx \gamma_{\sigma}^{-1} / 2$, and $R_{\sigma} \approx \gamma_{\sigma}^{-1 / 2} / 2$, with $\gamma_{\sigma}=\Gamma /(\Delta+\sigma h)$. Therefore the integrand scales as $\left(8 \gamma_{\sigma}\right)^{-3 / 2}$, whereas the width of the peak is $\propto \Gamma$. Overall, this means a diverging integral scaling like $\sim \Gamma^{-1 / 2}$. Similar divergence was found in Ref. [6] for the supercurrent-induced charge imbalance in the absence of spin splitting.

In practice, the relevant depairing mechanism in the presence of spin splitting and supercurrent is the orbital depairing due to the combined effect of the supercurrent itself and of an in-plane magnetic field $\boldsymbol{B}$ on the spectrum of the superconductor [35-37], with rate $\tau_{\text {orb }}^{-1}=D \Delta\left(\partial_{x} \varphi\right)^{2} /(2)+D e^{2} B^{2} d^{2} / 6$ for a film with thickness $d$. It does not relax the spin but affects the spectral properties of the superconductor by reshaping the singularities in the spectral quantities [3]. We can hence use $\tau_{\text {orb }}^{-1}$ instead of $\Gamma$ to cut the divergence and see that for very large phase gradients, $\mu_{z}$ becomes independent of $\partial_{x} \varphi$.

According to Eq. (16) the difference in the quantity $N_{\sigma}^{2} j_{s}^{\sigma} /\left(D_{L}^{\sigma} R_{T}^{\sigma}\right)$ for spin-up and -down species describes the charge-spin conversion. We find that the charge imbalance in each spin subband is proportional to the energy integral of $N_{\sigma}^{2} / R_{T}^{\sigma}$. The charge in each subband is then converted to spin at a rate $\propto j_{s}^{\sigma} / D_{L}^{\sigma}$. The temperature and exchange field dependence of $\mu_{z}$ are given in Figs. 3(c) and 3(d), respectively. We can see that the linear response $\mu_{z} \rightarrow 0$ as $T \rightarrow 0$, which reflects the freezing out of the quasiparticle population [Fig. 3(c)]. However, this can be circumvented by considering the response at $V_{\mathrm{inj}} \sim \Delta$, as shown below. At the superconducting critical temperature $T_{c}$, the ratio $\mu_{z} / I_{s}$ diverges similarly to the supercurrent-induced charge imbalance in the presence of a temperature gradient [7,8]. Since $T_{c}$ is lower for a higher exchange field, this divergence happens at a lower temperature in a higher exchange field. For a fixed temperature, the divergence of $\mu_{z}$ also happens at a critical exchange field [Fig. 3(d)] where superconductivity is suppressed [38,39].

\section{B. Effect of spin relaxation}

Spin-flip and spin-orbit relaxation affect both spectral and nonequilibrium properties of the superconductor. For the 
spectral properties, spin-flip relaxation breaks the timereversal symmetry and suppresses the superconducting pair potential and critical temperature, while spin-orbit scattering reduces the effect of the exchange field without suppressing the pair potential [3]. Both spin-flip and spin-orbit scattering also lead to the relaxation of $f_{T 3}\left[S_{T 3 / L 3}\right.$ terms in Eq. (6)]. For strong spin relaxation, the contribution to $\mu_{z}$ thus results only from $f_{L}$ and decays only via inelastic scattering. In this case (see details in Appendix C)

$$
\mu_{z}=\xi_{0} \partial_{x} \varphi_{0} \frac{G_{\mathrm{inj}}}{G_{\xi_{0}}} \int_{0}^{\infty} d \epsilon n_{-}\left(\epsilon ; V_{\mathrm{inj}}\right) \frac{\left(N_{\uparrow}^{2}-N_{\downarrow}^{2}\right) j_{E}}{4 R_{T} D_{L}} u_{1}(x) .
$$

Here the linear function $u_{1}(x)=-u_{1}(-x) \approx u_{0}(x)$ for $|x|>$ $\lambda_{\text {cr }}$. However, the effects of spin-flip/spin-orbit scattering on the spectral functions also affect the resulting $\mu_{z}$. The effect depends strongly on the type of scattering.

For pure spin-flip relaxation, contribution of $f_{L}$ increases as a function of the spin-relaxation rate and diverges when the strong relaxation completely kills superconductivity. This can be seen in the relaxation rate dependence of $\mu_{z}$ in the linearresponse regime in Fig. 3(b). For pure spin-orbit relaxation, the effect of the exchange field is suppressed, and thereby so is the charge-spin conversion.

\section{SPIN ACCUMULATION AND NONLOCAL CONDUCTANCE}

The charge-spin conversion can be detected by inspecting the nonlocal conductance $g_{\mathrm{nl}}=d I_{\mathrm{det}} / d V_{\mathrm{inj}}$ in the presence of the supercurrent $I_{s}$ driven across the wire. Without supercurrent, this quantity was measured in Refs. [25-27]. We show an example of $g_{\mathrm{nl}}$ in Figs. 4(c) and 4(d). We separate it into different symmetry components vs $V_{\text {inj }}$ and $P_{\text {det }}$ as

$$
g_{\mathrm{nl}}=g_{\mathrm{ee}}+g_{\mathrm{eo}}+\left(g_{\mathrm{oe}}+g_{\mathrm{oo}}\right) P_{\mathrm{det}},
$$

where $g_{\alpha e / o}\left(V_{\mathrm{inj}}\right)= \pm g_{\alpha e / o}\left(-V_{\mathrm{inj}}\right)$ and $\alpha=e / o$ describe the symmetry vs $P_{\text {det }}$. Since the derivative of the detector current with respect to $V_{\text {inj }}$ flips the parity of the terms, the conductance due to the pure charge imbalance is even in both $V_{\text {inj }}$ and $P_{\text {det }}$ and hence is described by $g_{\text {ee }}$. The term $g_{\text {oo }}=g_{\text {heat }}$ is the longrange spin accumulation due to the heat injection $[4,5]$. The supercurrent induces the term $g_{\text {eo }}$ that describes the conversion of temperature gradients to charge [6-8], whereas $g_{\text {oe }}=g_{\text {super }}$ results from the supercurrent-induced charge-spin conversion. The symmetry of $g_{\text {super }}$ results from the fact that it is related to spin imbalance (and therefore antisymmetric in $P_{\text {det }}$ ) and originates from induced charge imbalance. In normal-metal spin injection experiments [40] only the term $g_{\text {oe }}$ is nonzero, but it requires nonzero spin polarization $P_{\text {inj }}$ of the injector. Here $P_{\text {inj }}=0$.

The term $g_{\text {super }}$ should be compared to the contribution determined by effective heating [4] (14),

$$
g_{\text {heat }}=\frac{G_{\text {inj }}}{G_{\xi_{0}}} \frac{L}{2 \xi_{0}} u_{3}(x) \int_{0}^{\infty} d \epsilon \frac{\partial n_{+}}{\partial V_{\text {inj }}} \frac{N_{\uparrow}^{2}-N_{\downarrow}^{2}}{D_{L}},
$$

where $u_{3}(x)=u_{3}(-x)$ is a function that changes linearly from unity at the injector to zero at the reservoirs and $n_{+}=$ $\left(n_{0}(\epsilon+e V)+n_{0}(\epsilon-e V)-2 n_{0}\right) / 2$. For $T \rightarrow 0, \partial n_{ \pm} / \partial V_{\text {inj }}$ approaches a $\delta$ function at $\epsilon= \pm e V$, and we can esti- mate the integrals by the values of the kinetic coefficients at those energies. For $e V \approx \Delta \pm h$ where the main signal resides, $g_{\text {super }} \approx 2 \xi_{0} g_{\text {heat }} / L$ for $\xi_{0} \nabla \varphi \approx \tau_{\text {orb }}^{-1} \Delta+\tau_{\text {sf }}^{-1}+\tau_{\text {so }}^{-1}$, i.e., when the supercurrent starts affecting the density of states. At higher temperatures and lower voltages $e V \lesssim k_{B} T$, where quasiparticle effects are visible even at linear response, $g_{\text {super }}$ can dominate over $g_{\text {heat }}$

\section{CONCLUSION}

In conclusion, we have shown how the nonequilibrium supercurrent in a spin-split superconductor can partially convert charge imbalance to spin imbalance. The resulting spin imbalance is long range, decaying only due to inelastic scattering. Here we have concentrated on a setup with collinear magnetizations. We expect that the generalization of our theory to the case with inhomogeneous magnetization would shed light on the possible coherently controllable nonequilibrium spin torques. We also expect to find analogous effects in superconducting proximity structures in the presence of spin splitting, i.e., combining the phenomena discussed in Refs. [41,42].

\section{ACKNOWLEDGMENTS}

We thank M. Houzet and M. Aprili for the question that started this project and T. Hyart and C. Quay for illuminating discussions. This work was supported by the Academy of Finland Center of Excellence (Project No. 284594), Research Fellow (Project No. 297439), and Key Funding (Project No. 305256) programs.

\section{APPENDIX A: SELF-CONSISTENCY EQUATION FOR $\triangle$} from

The pair potential $\Delta$ should be obtained self-consistently

$$
\Delta=\frac{\lambda}{16} \int_{-\Omega_{D}}^{\Omega_{D}} d \epsilon \operatorname{Tr}\left[\left(\tau_{1}-i \tau_{2}\right) \hat{g}^{K}(\epsilon)\right],
$$

where $\lambda$ is the coupling constant and $\Omega_{D}$ is the Debye cutoff energy. In the presence of both spin splitting and nonequilibrium distribution functions, this goes to the form [3]

$$
\begin{aligned}
\Delta= & \frac{\lambda}{2} \int_{-\Omega_{D}}^{\Omega_{D}} d \varepsilon\left[\operatorname{Im} g_{01}^{R} f_{L}+\operatorname{Im} g_{31}^{R} f_{T 3}\right. \\
& \left.+i\left(\operatorname{Re} g_{01}^{R} f_{T}+\operatorname{Re} g_{31}^{R} f_{L 3}\right)\right],
\end{aligned}
$$

where $g_{i j}^{R}$ is the part of the retarded Green's function proportional to $\sigma_{i} \tau_{j}$. The results obtained in the main text use the self-consistent equilibrium gap but do not include the nonequilibrium corrections. For the gap amplitude $|\Delta|$ this approximation is justified in the case of low injection conductance $G_{\text {inj. }}$. However, with such a choice the charge current is, strictly speaking, not conserved in the presence of a constant phase gradient. This is because the quasiparticle injection modifies the phase of $\Delta$ [the last two terms in Eq. (A2)], and the true phase gradient corresponding to a constant charge current becomes position dependent. Such an effect is of a higher order in the phase gradient and within a perturbation approach can therefore be disregarded. We leave such higher-order effects for further work. 


\section{APPENDIX B: KINETIC COEFFICIENTS}

The Green's function in Eq. (2) satisfies the normalization condition $\breve{g}^{2}=1$, which allows us to parametrize the Keldysh Green's function as $\check{g}^{K}=\check{g}^{R} \check{f}-\check{f}^{\prime} \breve{g}^{A}$, where the distribution matrix $\check{f}=f_{L}+f_{T} \tau_{3}+f_{T 3} \sigma_{3}+f_{L 3} \sigma_{3} \tau_{3}$. We also can parametrize the retarded Green's function as $\check{g}^{R}=g_{01} \tau_{1}+g_{02} \tau_{2}+g_{03} \tau_{3}+g_{31} \sigma_{3} \tau_{1}+g_{32} \sigma_{3} \tau_{2}+$ $g_{33} \sigma_{3} \tau_{3}$, and $\check{g}^{A}=-\tau_{3} \breve{g}^{R \dagger} \tau_{3}$. Here $g_{i}$ are complex scalar functions. From these, we identify $N_{+}=\operatorname{Re}\left(g_{03}\right)$ and $N_{-}=$ $\operatorname{Re}\left(g_{33}\right)$.

The kinetic coefficients $D_{i}, R_{i}$, and $S_{i}$ in Eqs. (3) and (4) can be expressed in terms of the parameterized functions $\breve{g}^{R}$ and $\check{g}^{A} . D_{i}$ are

$$
\begin{aligned}
D_{L}= & \frac{D}{2}\left(1-\left|g_{01}\right|^{2}-\left|g_{02}\right|^{2}+\left|g_{03}\right|^{2}-\left|g_{31}\right|^{2}\right. \\
& \left.-\left|g_{32}\right|^{2}+\left|g_{33}\right|^{2}\right), \\
D_{T 3}= & -D\left[\operatorname{Re}\left(g_{01} g_{31}^{*}\right)+\operatorname{Re}\left(g_{02} g_{32}^{*}\right)-\operatorname{Re}\left(g_{03} g_{33}^{*}\right)\right], \\
D_{T}= & \frac{D}{2}\left(1+\left|g_{01}\right|^{2}+\left|g_{02}\right|^{2}+\left|g_{03}\right|^{2}+\left|g_{31}\right|^{2}\right. \\
& \left.+\left|g_{32}\right|^{2}+\left|g_{33}\right|^{2}\right), \\
D_{L 3}= & D\left[\operatorname{Re}\left(g_{01} g_{31}^{*}\right)+\operatorname{Re}\left(g_{02} g_{32}^{*}\right)+\operatorname{Re}\left(g_{03} g_{33}^{*}\right)\right] .
\end{aligned}
$$

$R_{i}$ are

$$
\begin{aligned}
R_{T} & =\operatorname{Re}\left(g_{01}\right) \Delta \cos \varphi-\operatorname{Re}\left(g_{02}\right) \Delta \sin \varphi, \\
R_{L 3} & =\operatorname{Re}\left(g_{31}\right) \Delta \cos \varphi-\operatorname{Re}\left(g_{32}\right) \Delta \sin \varphi .
\end{aligned}
$$

$S_{i}$ are

$$
\begin{aligned}
S_{L 3}= & \tau_{\mathrm{sn}}^{-1}\left\{\operatorname{Re}\left(g_{03}\right)^{2}-\operatorname{Re}\left(g_{33}\right)^{2}\right. \\
& \left.+\beta\left[\operatorname{Im}\left(g_{01}\right)^{2}-\operatorname{Im}\left(g_{31}\right)^{2}+\operatorname{Im}\left(g_{02}\right)^{2}-\operatorname{Im}\left(g_{32}\right)^{2}\right]\right\}, \\
S_{T 3}= & \tau_{\mathrm{sn}}^{-1}\left\{\operatorname{Re}\left(g_{03}\right)^{2}-\operatorname{Re}\left(g_{33}\right)^{2}\right. \\
& \left.+\beta\left[\operatorname{Re}\left(g_{31}\right)^{2}-\operatorname{Re}\left(g_{01}\right)^{2}+\operatorname{Re}\left(g_{32}\right)^{2}-\operatorname{Re}\left(g_{02}\right)^{2}\right]\right\},
\end{aligned}
$$

where $\tau_{\mathrm{sn}}^{-1}=\tau_{\mathrm{so}}^{-1}+\tau_{\mathrm{sf}}^{-1}$ and the parameter $\beta=\left(\tau_{\mathrm{so}}-\right.$ $\left.\tau_{\mathrm{sf}}\right) /\left(\tau_{\mathrm{so}}+\tau_{\mathrm{sf}}\right)$ describes the relative strength of the spinorbit and spin-flip scattering. For $\beta>0$, spin-flip scattering dominates the spin-orbit scattering and vice versa for $\beta<0$. These coefficients are independent of $\varphi$ (the dependence of $\varphi$ in $R_{i}$ terms is canceled by the corresponding terms in $g_{i}$ ).

There are also two more coefficients in Eqs. (3) and (4), spectral supercurrent and spectral spin supercurrent, which depend on the phase gradient $\partial_{x} \varphi$ :

$$
\begin{aligned}
& j_{E} \partial_{x} \varphi=\frac{1}{8} D \operatorname{Tr}\left[\left(\check{g}^{R} \partial_{x} \check{g}^{R}-\check{g}^{A} \partial_{x} \check{g}^{A}\right) \tau_{3}\right], \\
& j_{E s} \partial_{x} \varphi=\frac{1}{8} D \operatorname{Tr}\left[\left(\check{g}^{R} \partial_{x} \check{g}^{R}-\check{g}^{A} \partial_{x} \check{g}^{A}\right) \sigma_{3} \tau_{3}\right] .
\end{aligned}
$$

These two terms are related to the nonzero charge supercurrrent and spin-energy current. Here and below we assume that the wire is in the $x$ direction and all changes in the phase $\varphi$ and the distribution functions take place in that direction.

\section{APPENDIX C: PERTURBATION THEORY SOLUTIONS OF KINETIC EQUATIONS IN THE LINEAR ORDER BY $\xi_{0} \nabla \varphi$}

The general solution of the kinetic equations in Eq. (3) can be written as

$$
\begin{aligned}
& \left(f_{L}, f_{T 3}, f_{T}, f_{L 3}\right)^{T} \\
& =\left(C_{01}+C_{02} x\right) \mathbf{v}_{0}^{T}+C_{1} e^{k_{L} x} \mathbf{v}_{1}^{T}+C_{2} e^{-k_{L} x} \mathbf{v}_{2}^{T}+C_{3} e^{k_{T 1} x} \mathbf{v}_{3}^{T} \\
& \quad+C_{4} e^{-k_{T 1} x} \mathbf{v}_{4}^{T}+C_{5} e^{k_{T 2} x} \mathbf{v}_{5}^{T}+C_{6} e^{-k_{T 2} x} \mathbf{v}_{6}^{T},
\end{aligned}
$$

where $\mathbf{v}_{0}^{T}=(1,0,0,0)^{T} ; k_{L}, k_{T 1}$, and $k_{T 2}$ are the energydependent inverse length scales; the other $\mathbf{v}_{i}^{T}$ can be determined numerically; and $C_{i}$ can be determined from the boundary conditions (10). For a small phase gradient, we can determine these coefficients analytically. Below we concentrate in particular on the solutions of the modes related to the supercurrent-induced spin imbalance and treat the supercurrent as a perturbation in the kinetic equations. At zeroth order Eq. (3) decouples into two sets of kinetic equations. First, we concentrate on the part that is odd in the injection voltage, describing charge imbalance. In this case, for a vanishing supercurrent the relevant distribution function components are $f_{T}$ and $f_{L 3}$. We denote their values in the absence of supercurrent by $f_{T}^{0}$ and $f_{L 3}^{0}$. On the other hand, the supercurrent couples them to the other two functions $f_{L}$ and $f_{T 3}$ and induces the change $\delta f_{L}$ and $\delta f_{T 3}$, which we calculate to linear order in the phase gradient. For $f_{T}$ and $f_{L 3}$, we get the first set of kinetic equations,

$$
\left(\begin{array}{cc}
D_{T} & D_{L 3} \\
D_{L 3} & D_{T}
\end{array}\right)\left(\begin{array}{c}
\partial_{x}^{2} f_{T}^{0} \\
\partial_{x}^{2} f_{L 3}^{0}
\end{array}\right)=\left(\begin{array}{cc}
R_{T} & R_{L 3} \\
R_{L 3} & R_{T}+S_{L 3}
\end{array}\right)\left(\begin{array}{c}
f_{T}^{0} \\
f_{L 3}^{0}
\end{array}\right)
$$

In what follows, we choose $\Delta_{0}$ to be the reference energy scale, and therefore the coherence length $\xi_{0}=\sqrt{\hbar D / \Delta_{0}}$ becomes the reference length scale. That means, for example, that the dimensionless quantities describing spin relaxation are of the form $\tau_{\mathrm{sf}} \Delta_{0}$ and $\tau_{\mathrm{so}} \Delta_{0}$.

Using the boundary conditions (10), we obtain for $\kappa_{I} L \ll 1$

$$
\left(\begin{array}{c}
f_{T}^{0} \\
f_{L 3}^{0}
\end{array}\right)=\kappa_{I} \xi_{0} n_{-}\left(\epsilon, V_{\mathrm{inj}}\right) \sum_{i=1,2} A_{i} e^{-k_{T i} x / \xi_{0}}\left(\begin{array}{c}
k_{R_{i}} \\
-1
\end{array}\right), \quad 0 \leqslant x \leqslant \frac{L}{2},
$$

where the inverse length scales

$$
k_{T 1 / 2}^{2}=\frac{D_{T}\left(2 R_{T}-S_{L 3}\right)-2 D_{L 3} R_{L 3} \pm \sqrt{4\left(D_{T} R_{L 3}-D_{L 3} R_{T}\right)^{2}+4 D_{L 3}\left(-D_{T} R_{L 3}+D_{L 3} R_{T}\right) S_{L 3}+D_{T}^{2} S_{L 3}^{2}}}{2\left(D_{T}^{2}-D_{L 3}^{2}\right)},
$$

and the coefficients

$$
A_{i}=\frac{\left[N_{-}\left(D_{L 3}-D_{T} k_{R i^{\prime}}\right)-N_{+}\left(D_{T}-D_{L 3} k_{R i^{\prime}}\right)\right]}{4\left(D_{L 3}^{2}-D_{T}^{2}\right)\left(k_{R i}-k_{R i^{\prime}}\right) k_{T i}}
$$




$$
k_{R 1 / 2}=\frac{D_{T} S_{L 3} \mp \sqrt{4 D_{L 3}^{2} R_{T}\left(R_{T}+S_{L 3}\right)-4 D_{L 3} D_{T} R_{L 3}\left(2 R_{T}+S_{L 3}\right)+D_{T}^{2}\left(4 R_{L 3}^{2}+S_{L 3}^{2}\right)}}{2\left(D_{T} R_{L 3}-D_{L 3} R_{T}\right)} .
$$

For the perturbed terms of $f_{L}$ and $f_{T 3}$, we get another set of kinetic equations,

$$
\left(\begin{array}{cc}
D_{L} & D_{T 3} \\
D_{T 3} & D_{L}
\end{array}\right)\left(\begin{array}{c}
\partial_{x}^{2} \delta f_{L} \\
\partial_{x}^{2} \delta f_{T 3}
\end{array}\right)+\left(\begin{array}{cc}
j_{E} \partial_{x} \varphi & j_{E s} \partial_{x} \varphi \\
j_{E s} \partial_{x} \varphi & j_{E} \partial_{x} \varphi
\end{array}\right)\left(\begin{array}{c}
\partial_{x} f_{T}^{0} \\
\partial_{x} f_{L 3}^{0}
\end{array}\right)=\left(\begin{array}{cc}
0 & 0 \\
0 & S_{T 3}
\end{array}\right)\left(\begin{array}{c}
\partial_{x}^{2} \delta f_{L} \\
\partial_{x}^{2} \delta f_{T 3}
\end{array}\right) .
$$

Using the solution in Eq. (C3), we obtain

$$
\begin{aligned}
\left(\begin{array}{c}
\delta f_{L} \\
\delta f_{T 3}
\end{array}\right)= & \kappa_{I} \xi_{0}^{2} \partial_{x} \varphi n_{-}\left(\epsilon, V_{\mathrm{inj}}\right) \sum_{i=1,2}\left[\frac{\alpha_{i}}{k_{L}^{2}-k_{T i}^{2}}\left(e^{-k_{T i} x / \xi_{0}}-e^{-k_{L} x / \xi_{0}}\right)\left(\begin{array}{c}
-D_{T 3} / D_{L} \\
1
\end{array}\right)\right. \\
& \left.+\frac{\beta_{i}}{k_{T i}^{2}}\left(\frac{2 x}{L}-1+e^{-k_{T i} x / \xi_{0}}\right)\left(\begin{array}{l}
1 \\
0
\end{array}\right)\right], \quad 0 \leqslant x \leqslant \frac{L}{2},
\end{aligned}
$$

where the inverse length scale

$$
k_{L}^{2}=\frac{S_{T 3} D_{L}}{D_{L}^{2}-D_{T 3}^{2}}
$$

and the coefficients

$$
\begin{gathered}
\alpha_{i}=\frac{\left[j_{E s}\left(D_{T 3}+D_{L} k_{R i}\right)-j_{E}\left(D_{L}+D_{T 3} k_{R i}\right)\right]\left[N_{-}\left(D_{L 3}-D_{T} k_{R i^{\prime}}\right)-N_{+}\left(D_{T}-D_{L 3} k_{R i^{\prime}}\right)\right]}{2\left(D_{T}^{2}-D_{L 3}^{2}\right)\left(D_{L}^{2}-D_{T 3}^{2}\right)\left(k_{R i}-k_{R i^{\prime}}\right)}, \\
\beta_{i}=\frac{\left(j_{E} k_{R i}-j_{E s}\right)\left[N_{+}\left(D_{T}-D_{L 3} k_{R i^{\prime}}\right)-N_{-}\left(D_{L 3}-D_{T} k_{R i^{\prime}}\right)\right]}{2 D_{L}\left(D_{T}^{2}-D_{L 3}^{2}\right)\left(k_{R i}-k_{R i^{\prime}}\right)}
\end{gathered}
$$

The spin accumulation generated from the supercurrent is

$$
\begin{aligned}
\mu_{z}= & \frac{1}{2} \int_{0}^{\infty} d \epsilon\left(N_{+} \delta f_{T 3}+N_{-} \delta f_{L}\right) \\
= & \frac{1}{2} \kappa_{I} \xi_{0}^{2} \partial_{x} \varphi \int_{0}^{\infty} d \epsilon n_{-}\left(\epsilon, V_{\mathrm{inj}}\right) \sum_{i=1,2}\left[\left(N_{+}-N_{-} \frac{D_{T 3}}{D_{L}}\right) \frac{\alpha_{i}}{k_{L}^{2}-k_{T i}^{2}}\left(e^{-k_{T i} x / \xi_{0}}-e^{-k_{L} x / \xi_{0}}\right)\right. \\
& \left.+N_{-} \frac{\beta_{i}}{k_{T i}^{2}}\left(\frac{2 x}{L}-1+e^{-k_{T i} x / \xi_{0}}\right)\right], 0 \leqslant x \leqslant \frac{L}{2} .
\end{aligned}
$$

In the extreme limit of $\tau_{\mathrm{sn}}^{-1} \rightarrow 0$, this result can be reduced to a simpler form. In this case, $S_{T 3}$ and $S_{L 3}$ terms in the kinetic equations are zero; therefore the $e^{-k_{L} x / \xi_{0}}$ term is replaced by a linear term with the same coefficients with $\delta f_{L}$. For the linear-response regime $n_{-}\left(\epsilon, V_{\text {inj }}\right)=V_{\text {inj }} \partial n_{0} / \partial \epsilon$, we get

$$
\begin{aligned}
\mu_{z}= & V_{\mathrm{inj}} \kappa_{I} \xi_{0}^{2} \partial_{x} \varphi \int_{0}^{\infty} d \epsilon \frac{\partial n_{0}}{\partial \epsilon}\left[\frac{N_{\uparrow}^{2} j_{s}^{\uparrow}}{4 D_{L}^{\uparrow} R_{T}^{\uparrow}}\left(\frac{2 x}{L}-1+e^{-\sqrt{R_{T}^{\uparrow} / D_{T}^{\uparrow}} x / \xi_{0}}\right)\right. \\
& \left.-\frac{N_{\downarrow}^{2} j_{s}^{\downarrow}}{4 D_{L}^{\downarrow} R_{T}^{\downarrow}}\left(\frac{2 x}{L}-1+e^{-\sqrt{R_{T}^{\downarrow} / D_{T}^{\downarrow}} x / \xi_{0}}\right)\right], \quad 0 \leqslant x \leqslant \frac{L}{2},
\end{aligned}
$$

where the $\uparrow$ and $\downarrow$ quantities are the addition and subtraction of the singlet and triplet components of the spectral quantities, $j_{s}^{\uparrow / \downarrow}=j_{E} \pm j_{E s}, N_{\uparrow / \downarrow}=N_{+} \pm N_{-}, D_{L}^{\uparrow / \downarrow}=D_{L} \pm D_{T 3}$, and $R_{\uparrow / \downarrow}=R_{T} \pm R_{L 3}$.

It is straightforward to see that $\mu_{z}=0$ for $h=0$ since the quantity $N^{2} j_{s} /\left(D_{L} R_{T}\right)$ is equal for both spin species. For nonzero $h$ the difference in this quantity for different spin species gives the spin accumulation. However, without relax- ation, this quantity is proportional to $1 / \sqrt{\Gamma}$, which describes the broadening of the spectral quantities.

In practice, the relevant broadening renormalizing $\mu_{z}$ comes from the orbital effect due to either a magnetic field or 

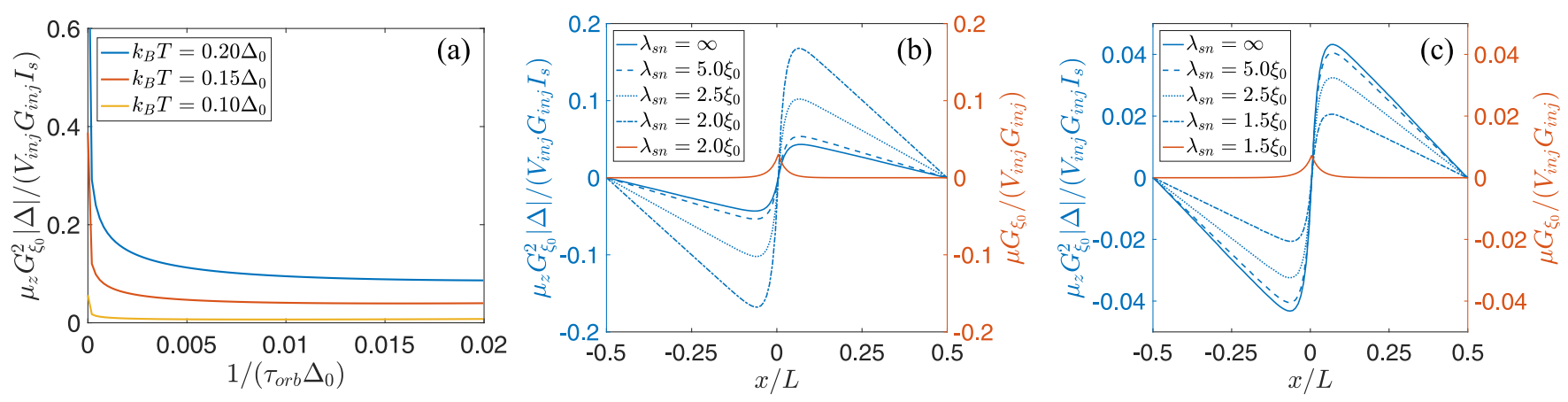

FIG. 5. Spin accumulation with and without relaxation in the linear-response regime. (a) The dependence on orbital depairing rate in the case without spin relaxation. Position dependence in the case of (b) pure spin-flip relaxation and (c) pure spin-orbit relaxation. An exchange field $h=0.3 \Delta_{0}$ is the same for all panels, and a temperature $T=0.15 \Delta_{0}$ is used in (b) and (c). The red curves describe the charge imbalance. The spin-relaxation length is defined as $\lambda_{\mathrm{sn}}=\sqrt{\tau_{\mathrm{sn}} D}$.

the phase gradient itself [35-37] or due to terms contributing to the spin relaxation [3]. The two first effects can be described by an orbital relaxation rate $\tau_{\text {orb }}^{-1}=\left(\xi_{0} \partial_{x} \varphi\right)^{2} / 2+$ $\left(D e^{2} B^{2} d^{2} / 6\right)$ [37], where $B$ is the magnetic field and $d$ is the film thickness. In the presence of spin relaxation described by the rate $\tau_{\mathrm{sn}}^{-1}$, an estimate for the overall broadening comes from $\Gamma \mapsto \tau_{\mathrm{orb}}^{-1}+\tau_{\mathrm{sn}}^{-1}$, but the exact amount depends on the relaxation mechanism and the size of the exchange field. As an example, we show the supercurrentinduced $\mu_{z}$ vs $\tau_{\text {orb }}^{-1}$ in Fig. 5(a). Since $\mu_{z} \propto\left(\xi_{0} \partial_{x} \varphi\right) \Gamma^{-1 / 2}$, for large phase gradients satisfying $\xi_{0} \partial_{x} \varphi \gg \sqrt{D e^{2} B^{2} d^{2} / 6}+$ $\tau_{\mathrm{sn}}^{-1}$, the spin accumulation becomes independent of $\partial_{x} \varphi$.

However, spin relaxation also affects the decay of the nonequilbrium components of the distribution function via the relaxation terms $\sim S_{T / L 3}$. In another extreme limit $\tau_{\mathrm{sn}} \rightarrow \infty$, we can also have a simpler form of Eq. (C7). In this case

$$
\begin{aligned}
4 D_{L 3}( & \left.D_{T} R_{L 3}-D_{L 3} R_{T}\right) / D_{T}^{2} \ll S_{L 3} \text {, and } \\
\mu_{z}= & V_{\mathrm{inj}} \kappa_{I} \xi_{0}^{2} \partial_{x} \varphi \int_{0}^{\infty} d \epsilon \frac{\partial n_{0}}{\partial \epsilon} \frac{\left(N_{\uparrow}^{2}-N_{\downarrow}^{2}\right) j_{E}}{4 R_{T} D_{L}} \\
& \times\left(\frac{2 x}{L}-1+2 e^{-k_{T 2} x / \xi_{0}}-e^{-k_{L} x / \xi_{0}}\right), \quad 0 \leqslant x \leqslant \frac{L}{2} .
\end{aligned}
$$

Here, except for the density of states, the triplet component of other spectral quantities do not contribute to the spin accumulation. The difference in the density of states for two spin species behaves differently for spin-orbit and spin-flip relaxations. Spin-orbit relaxation does not affect the pair potential but tries to lift the effect of the spin-splitting field. Therefore $\mu_{z}$ approaches zero for very strong relaxation [Fig. 5(c)]. In the case of spin-flip relaxation, it suppresses the pair potential; therefore spin accumulation diverges [Fig. 5(b)].
[1] M. Tinkham, Introduction to Superconductivity (Dover Publications, Mineola, New York, 2004).

[2] A. Schmid and G. Schön, J. Low Temp. Phys. 20, 207 (1975).

[3] F. S. Bergeret, M. Silaev, P. Virtanen, and T. T. Heikkilä, Rev. Mod. Phys. (to be published), arXiv:1706.08245.

[4] M. Silaev, P. Virtanen, F. S. Bergeret, and T. T. Heikkilä, Phys. Rev. Lett. 114, 167002 (2015).

[5] I. V. Bobkova and A. M. Bobkov, JETP Lett. 101, 118 (2015).

[6] A. Schmid and G. Schön, Phys. Rev. Lett. 43, 793 (1979).

[7] J. Clarke, B. R. Fjordbøge, and P. E. Lindelof, Phys. Rev. Lett. 43, 642 (1979).

[8] C. Pethick and H. Smith, J. Phys. C 13, 6313 (1980).

[9] C. J. Pethick and H. Smith, Phys. Rev. Lett. 43, 640 (1979).

[10] V. M. Edelstein, Phys. Rev. Lett. 75, 2004 (1995).

[11] V. M. Edelstein, Phys. Rev. B 67, 020505 (2003).

[12] V. M. Edelstein, Phys. Rev. B 72, 172501 (2005).

[13] V. Edel'shtein, Zh. Eksp. Teor. Fiz. 95, 2151 (1989) [Sov. Phys. JETP 68, 1244 (1989)].

[14] M. Houzet and J. S. Meyer, Phys. Rev. B 92, 014509 (2015).

[15] K. V. Samokhin, Phys. Rev. B 70, 104521 (2004).

[16] O. Dimitrova and M. V. Feigel'man, Phys. Rev. B 76, 014522 (2007)

[17] A. Buzdin, Phys. Rev. Lett. 101, 107005 (2008).
[18] F. Konschelle, I. V. Tokatly, and F. S. Bergeret, Phys. Rev. B 92, 125443 (2015).

[19] I. V. Bobkova, A. M. Bobkov, A. A. Zyuzin, and M. Alidoust, Phys. Rev. B 94, 134506 (2016).

[20] I. V. Bobkova and A. M. Bobkov, Phys. Rev. B 95, 184518 (2017).

[21] D. B. Szombati, S. Nadj-Perge, D. Car, S. R. Plissard, E. P. A. M. Bakkers, and L. P. Kouwenhoven, Nat. Phys. 12, 568 (2016).

[22] A. Assouline, C. Feuillet-Palma, N. Bergeal, T. Zhang, A. Mottaghizadeh, A. Zimmers, E. Lhuillier, M. Marangolo, M. Eddrief, P. Atkinson et al., arXiv:1806.01406.

[23] A. Murani, A. Kasumov, S. Sengupta, Y. A. Kasumov, V. T. Volkov, I. I. Khodos, F. Brisset, R. Delagrange, A. Chepelianskii, R. Deblock, H. Bouchiat, and S. Guéron, Nat. Commun. 8, 15941 (2017).

[24] Y. K. Kato, R. C. Myers, A. C. Gossard, and D. D. Awschalom, Phys. Rev. Lett. 93, 176601 (2004).

[25] C. Quay, D. Chevallier, C. Bena, and M. Aprili, Nat. Phys. 9, 84 (2013).

[26] F. Hübler, M. J. Wolf, D. Beckmann, and H. v. Löhneysen, Phys. Rev. Lett. 109, 207001 (2012).

[27] M. J. Wolf, F. Hübler, S. Kolenda, H. v. Löhneysen, and D. Beckmann, Phys. Rev. B 87, 024517 (2013). 
[28] F. S. Bergeret, A. Volkov, and K. Efetov, Rev. Mod. Phys. 77, 1321 (2005).

[29] T. T. Heikkilä, J. Särkkä, and F. K. Wilhelm, Phys. Rev. B 66, 184513 (2002).

[30] M. Y. Kuprianov and V. Lukichev, Zh. Eksp. Teor. Fiz. 94, 139 (1988) [Sov. Phys. JETP 67, 1163 (1988)].

[31] F. S. Bergeret, A. Verso, and A. F. Volkov, Phys. Rev. B 86, 214516 (2012).

[32] M. Tinkham and J. Clarke, Phys. Rev. Lett. 28, 1366 (1972).

[33] F. Hübler, J. C. Lemyre, D. Beckmann, and H. v. Löhneysen, Phys. Rev. B 81, 184524 (2010).

[34] R. C. Dynes, J. P. Garno, G. B. Hertel, and T. P. Orlando, Phys. Rev. Lett. 53, 2437 (1984).
[35] P. G. de Gennes, Superconductivity of Metals and Alloys, Advanced Book Classics (Perseus, Cambridge, MA, 1999).

[36] W. Belzig, C. Bruder, and G. Schön, Phys. Rev. B 54, 9443 (1996).

[37] A. Anthore, H. Pothier, and D. Esteve, Phys. Rev. Lett. 90, 127001 (2003).

[38] B. S. Chandrasekhar, Appl. Phys. Lett. 1, 7 (1962).

[39] A. M. Clogston, Phys. Rev. Lett. 9, 266 (1962).

[40] F. J. Jedema, A. Filip, and B. Van Wees, Nature (London) 410, 345 (2001).

[41] P. Virtanen and T. T. Heikkilä, Phys. Rev. Lett. 92, 177004 (2004).

[42] P. Machon, M. Eschrig, and W. Belzig, Phys. Rev. Lett. 110, 047002 (2013). 